\title{
sciendo
}

\section{Is conventionally calculated anchor-based minimum clinically important difference value catches the real clinical increment? Determining the situations that make the answer "no" by a simulation study}

\author{
Selcen YÜKSEL \\ Ankara Yildirim Beyazit University, Ankara, Turkey \\ selcenpehlivan@gmail.com \\ Pervin DEMİR \\ Ankara Yildirim Beyazit University, Ankara, Turkey \\ pervin.demr@gmail.com \\ Afra ALKAN \\ Ankara Yildirim Beyazit University, Ankara, Turkey \\ afra.alkan@gmail.com
}

\begin{abstract}
The aim of this study was to examine the accuracy of conventionally used method-optimal cutoff of Receiver Operating Characteristic (ROC) curve- to determine the minimum clinically important difference (MCID), which is the estimator of responsiveness for scales, by a simulation study. The baseline person parameters were firstly generated and, by using these values, two gold standard groups were constructed as "improved" and "non-improved" after the treatment. Five point-likert response patterns were obtained for 20 items in each group, representing pre-and post-treatment responses of individuals. The mean change score between post treatment and baseline scores for the improved group was considered as real MCID $\left(M C I D_{R}\right)$, after baseline and post-treatment total scores were calculated from response patterns. The cut-off for change score specified by ROC analysis, which best discriminates between improved group and not improved group, $M C I D_{R O C}$, was compared to $M C I D_{R}$. The scenarios of simulation were consisted of sample size and distribution of total scores for improved group. The data were generated for each of 40 scenarios with 1000 MCMC repeats. It was observed that the MCID ${ }_{R}$ and $M C I D_{R O C}$ were not so affected by sample size. However, MCID ${ }_{R O C}$ overestimated the $M C I D_{R}$ values in all scenarios. Briefly, the cut-off points obtained by ROC analysis found to be greater than the real MCID values. Therefore, alternative methods are required to calculate MCID.
\end{abstract}

Keywords: minimum clinically important difference, ROC, simulation, balanced design, unbalanced design

\section{Introduction}

A scale can be defined as a tool that is constructed to measure a specific feature and has proved psychometric characteristics (Yüksel, 2012). It is important to recognize whether the change in total score of any scale is perceived by patients as the magnitude of, which is an indicator of the scale's responsiveness. The statistical way of revealing whether the perceived change- difference between postop and baseline total scale score-is clinically important, is determining the minimum clinically important difference (MCID). MCID, the mean change 
score of patients who improves compared to baseline, was first defined as "the smallest difference in score in the domain of interest which patients perceive beneficial" by Jaeschke (Jaeschke, 1989)

Two defined MCID definition are used for calculation-anchor-based and distribution based MCID. Distribution-based or data driven approaches depend on the statistical characteristics of the data. In contrast, anchor-based approaches link the change in the outcome measure to a meaningful external anchor that accounts for the patient's perspective. For example, patients were asked to rate themselves according to their last condition state from "much worse," "slightly worse," "same," "slightly better" to "much better." The MCID for improvement can be defined as the mean difference of the outcome measures of patients who rated themselves "same" and "slightly better." But the problem here is that the patient should answer the question by comparing with his / her previous situation. It seems difficult in practice for the patient to perceive the question in that way and to respond according to theory.

One of the anchor-based approaches is to estimate MCID by the optimal cut-off point for change score between postop and baseline total scale scores, as a result of ROC Analysis. The ROC curve is a plot of the true positive fraction (TPF) and the false positive fraction (FPF) for all possible cut-point values of any diagnostic test (Pepe, 2003). The gold standard for ROC analysis is specified by an anchor question addressed after the treatment named "anchor question".

The major point that motivated us to do this work was the lack of the theoretical definition of ROC analysis, which was used to assess the accuracy of diagnostic tests, that could question the clinically significant improvement. So, the aim of the present study is to examine the accuracy of the cut-off point to determine the MCID by a simulation study with 1000 MCMC repeats in each 40 scenarios. The data were generated by using irr, moments and OptimalCutpoints packages in R Software (R Development Core Team (2008). R: A language and environment for statistical computing. R Foundation for Statistical Computing, Vienna, Austria. ISBN 3-900051-07-0, URL http://www.R-project.org.)

\section{Literature review}

The anchor-based and distribution-based approaches are widely used to calculate MCID among the several statistical methods (Group ISS,2013; Copay,2008; Crawford, 2015;Gum, 2013; Bago, 2009; Wells, 2001;.Yüksel, 2019) One of the anchor-based approaches is to estimate MCID by the optimal cut-off point for change score between post treatment and baseline scores, resulted from ROC Analysis. The gold standard for ROC analysis is specified by an anchor question addressed after the treatment. This question determines the patient progress during the treatment. "How has spine surgery changed your back status comparing to your baseline status?" can be given as an example. The anchor question generally has response categories between $[-7,+7]$. In the literature, the patients are divided into two groups as the patients responding at least "-2" and those responding any category less than "-2" and defined as "improved" and "not improved", respectively. The best cut-off point for change score is calculated by considering these groups as gold standard.

Yüksel, et al. have calculated the MCID values for some scales used to define health quality for adult spine deformity, from a new perspective using latent class analysis. In this study, instead of grouping individuals as improved and non-improved with a single anchor question, MCID values were obtained by modeling the response patterns they gave to each 
item in the scale. (Yüksel, 2019)

\section{Methodology}

Determination of the MCID is critical for judging whether treatments have resulted in real change and the magnitude of the benefit of scales. The MCID is the smallest meaningful change in score considered clinically important and constitutes a score difference related to a meaningful and beneficial change in health status perceived by the patient. There are two methods in the context of MCID: Distribution-based method and anchor-based method. Distribution based method is based on the statistical distributions of change scores and proportions of the pooled standard deviation ( $\mathrm{SD}_{\text {pooled}}$ ). Anchor-based method is the outcome measure of change onto an anchor criterion of change that is considered important to patients or clinicians. Reporting MCID values will provide information about important benchmarks for whether the observed changes can be considered reliable and clinically important. In this study anchor-based MCID was evaluated by ROC analysis perspective by calculating optimal cut-off point for change score between postop and baseline total scale scores.

One of the most commonly used methods for evaluating the performance of diagnostic tests with continuous response is the ROC analysis. The ROC analysis provides a mathematical approach to the identification of the accuracies of diagnostic tests. These curves show the relationship between the sensitivity and selectivity of the diagnostic test. When the diagnostic test result is continuous, an infinite number of critical values that can be taken as a cut-off point. ROC curves can be drawn with the help of varying sensitivity and selectivity values at different cut points. ROC curves are plotted for different cutting points by combining the sensitivity values (TPF) in the vertical axis corresponding to the false positive ratio (1-specificity) (FPF) values in the horizontal axis.

Let $\mathrm{X}$ be a continuous difference between postop and baseline total score which is assumed to be related to the true disease status, where $D$ and $\bar{D}$ present the presence and the absence of the disease, respectively. In practice, to calculate MCID, an anchor question is asked to patient and want him/her to rate his/her thereupon health status from -7 to +7 while comparing before treatment. Then a cut off value is determined on this difference (generally 0 as midpoint). Patients have value lower than 0 is defined as improved and vice versa as unimproved. After dichotomizing patients as "improved" and "unimproved", this new variable is defined as gold standard for ROC analysis.

The true positive fraction $\operatorname{TPF}(\mathrm{c})$ and the false positive fraction $\operatorname{FPF}(\mathrm{c})$ are respectively defined, at any given possible cut-off point c of $\mathrm{X}$, as

and

$$
T P F(c)=P(X>c \mid D)=S_{D}(c)
$$

$$
F P F(c)=P(X>c \mid \bar{D})=S_{\bar{D}}(c)
$$

\section{Youden Index method (J):}

The Youden index (J) (Youden, 1950) is the maximum achievable value of the Youden function $J(c)$, defined as the difference between the population quantities TPF(c) and FPF(c)

$$
J(c)=S_{D}(c)-S_{\bar{D}}(c)
$$

The optimal cut-off point $\hat{c}_{J}$ is the $\mathrm{c}$ that achieves the maximum of the Youden Function 
$\hat{J}(c)$ over all possible cut-off possible values of $X$.

As a result, the optimal cut-off value got from Youden index evaluated as MCID value for this study design.

\section{Simulation design}

The baseline person parameters are generated according to and sample size and improvement group size proportion within total sample size as stated at Table-1. The "improved" and "not improved" groups are constructed by adding and subtracting random positive numbers from uniform distribution to/from these parameters, thus the gold standard groups are acquired. Five-point-likert response patterns for baseline, "improved" and "not improved" groups are obtained by using Partial Credit Model with person parameters from the first step, for 20 items with item parameters being in the interval $[-7,+7]$. The total scores are calculated via response patterns. The mean change score between post-treatment and baseline scores of "improved" groups is set as real MCID $\left(M_{C} D_{R}\right)$. MCID $D_{R O C}$ is calculated by the cut-off point for change score that best discriminates between the "improved" and "not improved" groups determined by ROC analysis. MCID values from two methods are compared. The data are generated with 1000 MCMC repeats for each scenario.

Table 1. Simulation Scenarios

\begin{tabular}{|l|l|}
\hline Sample Size & $30 ; 50 ; 100 ; 150 ; 200 ; 300 ; 400 ; 500$ \\
\hline Improvement group size proportion within total sample size & $0.30 ; 0.40 ; 0.50 ; 0.60 ; 0.70$ \\
\hline
\end{tabular}

\section{Results and discussions}

It is clear that the median MCID values obtained by the ROC analysis (MCIDRoc)in Figure-1 and Table- 2 are higher than the median MCID values calculated by the average of the differences within improved group that defined as real MCID (MCIDR). From Table-2, Figure2 and Figure-3 one can concluded that MCIDRoc values are affected by the sample size and improvement group size proportion a little bit. Also, when sample size increases MCIDROC values decreases to get closed to MCIDR. In the same graphs, it is clear that the median

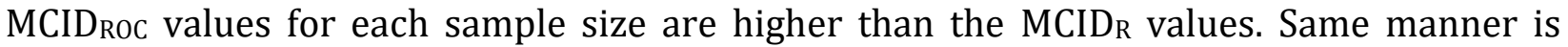
relevant for group proportion. While group design gets unbalanced, difference between $\mathrm{MCID}_{\mathrm{ROC}}$ and $\mathrm{MCID}_{\mathrm{R}}$ increases.

Table 2. Simulation results

\begin{tabular}{|r|l|l|}
\hline & MCID $_{\mathrm{R}}($ Median-Min-Max-IQR) & MCID $_{\text {Roc }}($ Median-Min-Max-IQR) \\
\hline \multicolumn{1}{|l|}{ Overall } & $20.96-7.55-37.88-2.28$ & $41.00-33.00-67.00-3.00$ \\
\hline Sample size & & \\
\hline 30 & $20.80-7.55-37.88-5.41$ & $44.00-35.00-67.00-6.00$ \\
\hline 50 & $20.80-9.06-35.00-4.00$ & $43.00-33.00-62.00-4.00$ \\
\hline 100 & $20.97-13.40-31.03-2.96$ & $42.00-35.00-57.00-3.00$ \\
\hline 150 & $20.99-13.53-28.44-2.35$ & $41.00-36.00-51.00-3.00$ \\
\hline 200 & $20.98-15.30-27.31-2.05$ & $41.00-35.00-47.00-2.00$ \\
\hline 300 & $20.97-15.58-25.93-1.75$ & $41.00-35.00-47.00-2.00$ \\
\hline 400 & $21.01-17.20-25.18-1.49$ & $41.00-37.00-46.00-2.00$ \\
\hline
\end{tabular}




\begin{tabular}{|c|c|c|}
\hline & MCIDR (Median-Min-Max-IQR) $^{2}$ & MCID $_{\text {Rос }}$ (Median-Min-Max-IQR) \\
\hline Overall & $20.96-7.55-37.88-2.28$ & $41.00-33.00-67.00-3.00$ \\
\hline 500 & $20.97-16.36-24.81-1.38$ & $41.00-37.00-46.00-2.00$ \\
\hline $\begin{array}{l}\text { Improvement } \\
\text { group size } \\
\text { proportion }\end{array}$ & & \\
\hline 0.3 & $20.91-7.55-37.88-2.91$ & $41.00-33.00-67.00-2.00$ \\
\hline 0.4 & $20.96-8.08-36.91-2.53$ & $41.00-35.00-64.00-2.00$ \\
\hline 0.5 & $20.96-10.84-32.73-2.23$ & $41.00-35.00-63.00-3$ \\
\hline 0.6 & $20.97-10.61-32.77-2.01$ & $42.00-35.00-64.00-2.00$ \\
\hline 0.7 & $20.97-12.00-32.04-1.85$ & $42.00-35.00-60.00-2.00$ \\
\hline
\end{tabular}

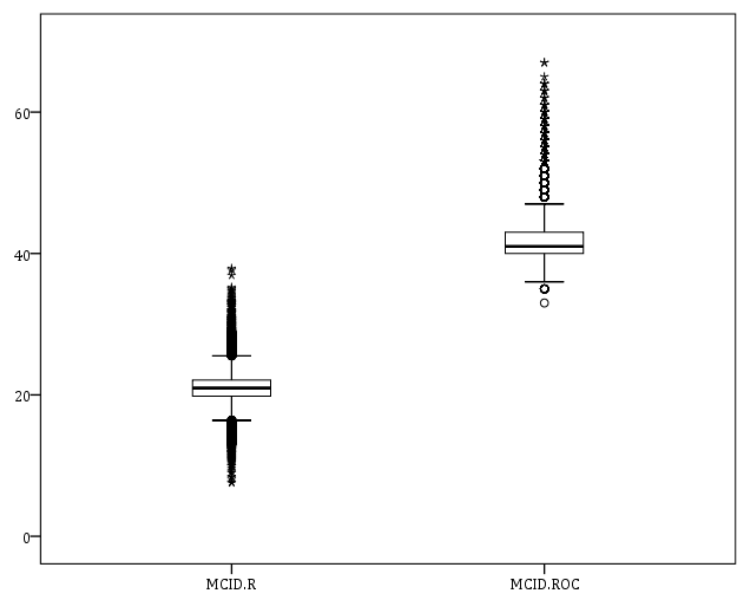

Figure 1. Summary of results of 400000 repeat

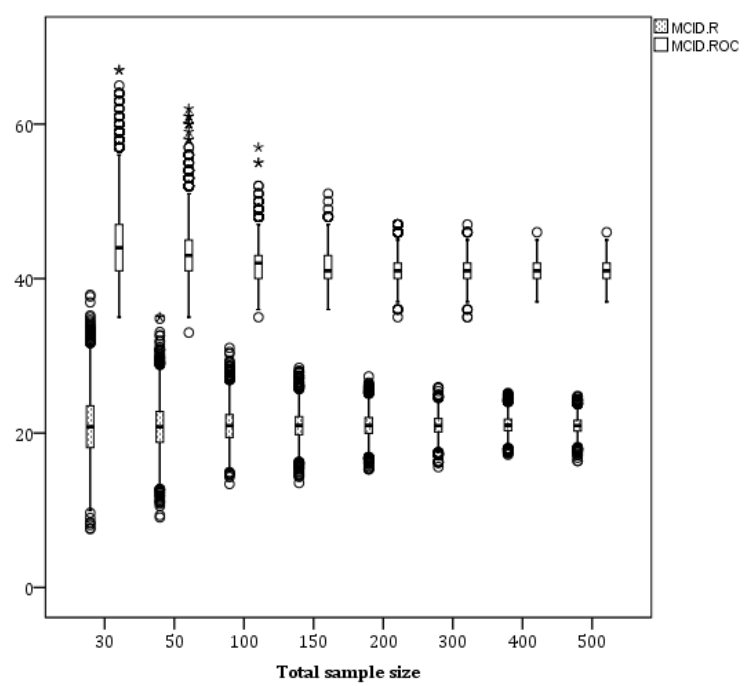

Figure 2. Summary of results for each total sample size 


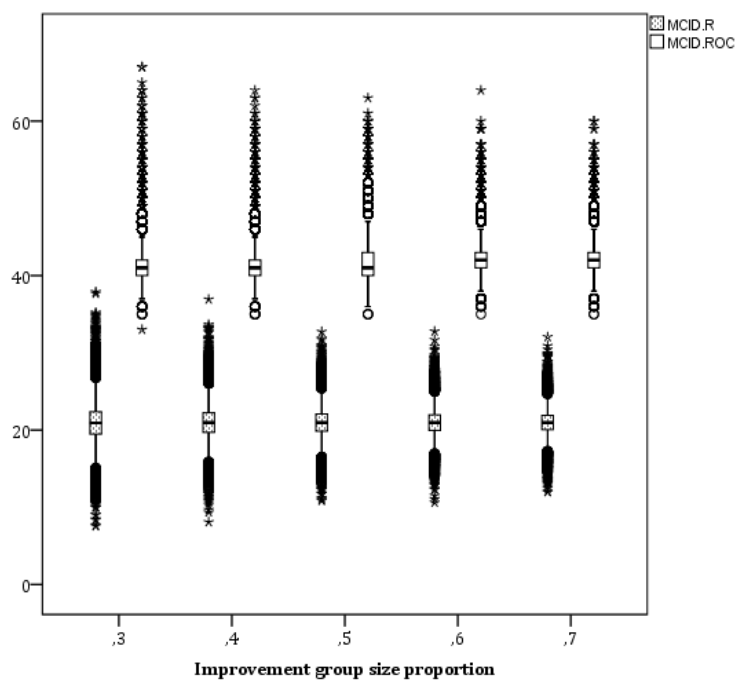

Figure 3. Summary of results for each improvement group size proportion

\section{Conclusion}

The aim of this study was to show how far the best cut-off value obtained by the ROC analysis from the real value calculated for the minimum clinically importance of difference in evaluating the interpretability of the scales. For this purpose, data on 40 different simulation scenarios were derived and the results were evaluated. In general, it is observed that the optimal cut-point on ROC curve overestimates MCID values. When each sample size and improvement group size proportion were taken into consideration, the same results were achieved. It was also observed that the result did not change as the sample size increased. In literature, $80 \%$ of the scale studies includes the MCID values calculated by the ROC analysis. The fact that the MCIDroc values are higher than the MCIDr is the proof that the ROC analysis will have biased results in the calculations on MCID. There is a need for advanced designs that question the interchangeability of results in different simulation conditions.

\section{References}

Andriessen, D. (2006). On the metaphorical nature of intellectual capital: a textual analysis. Journal of Intellectual Capital, 7(1), 93-110.

Bago J, Perez-Grueso FJ, Les E, Hernandez P, Pellise F. (2009) Minimal important differences of the SRS-22 Patient Questionnaire following surgical treatment of idiopathic scoliosis. European Spine Journal: official publication of the European Spine Society, the European Spinal Deformity Society, and the European Section of the Cervical Spine Research Society. ;18(12):1898-1904.

Copay AG, Glassman SD, Subach BR, Berven S, Schuler TC, Carreon LY. (2008) Minimum clinically important difference in lumbar spine surgery patients: a choice of methods using the Oswestry Disability Index, Medical Outcomes Study questionnaire Short Form 36, and pain scales. The Spine Journal: official journal of the North American Spine Society. ;8(6):968-974.

Crawford CH, 3rd, Glassman SD, Bridwell KH, Berven SH, Carreon LY. (2015) The minimum clinically important difference in SRS-22R total score, appearance, activity and pain domains after surgical treatment of adult spinal deformity. Spine. ;40(6):377-381.

Group ISS, McCarthy I, Bess S, et al. (2013) Calculating and Defining Minimally Important Clinical Difference (MCID) and Substantial Clinical Benefit (SCB) Values for Adult Spinal Deformity (ASD): A Robust Methodology for Consistent Data Reporting. The 
Spine Journal. ;13(9): S75.

Gum JL, Glassman SD, Carreon LY. (2013) Clinically important deterioration in patients undergoing lumbar spine surgery: a choice of evaluation methods using the Oswestry Disability Index, 36-Item Short Form Health Survey, and pain scales: clinical article. Journal of Neurosurgery Spine. ;19(5):564-568.

Jaeschke R, Singer J, Guyatt GH. (1989) Measurement of health status. Ascertaining the minimal clinically important difference. Controlled Clinical Trials. ;10(4):407-415.

Pepe MS. (2003). "The Statistical Evaluation of Medical Tests for Classification and Prediction." Oxford University Press: New York.

R Core Team (2008). R: A language and environment for statistical computing. R Foundation for Statistical Computing, Vienna, Austria. URL https://www.R-project.org/.

Wells G, Beaton D, Shea B, et al. (2001) Minimal clinically important differences: review of methods. The Journal of Rheumatology. ;28(2):406-412.

Youden WJ. (1950). "Index for rating diagnostic tests." Cancer, 3:32-35.

Yüksel S, Ayhan S, Nabiyev V, Domingo-Sabat M, Vila-Casademunt A, Obeid I, Perez-Grueso FS, Acaroglu E; European Spine Study Group (ESSG). (2019) Minimum clinically important difference of the health-related quality of life scales in adult spinal deformity calculated by latent class analysis: is it appropriate to use the same values for surgical and nonsurgical patients? Spine Journal; doi: 10.1016/j.spinee.07.005.

Yüksel,S. (2012) Ölçeklerde Saptanan Madde İşlev Farklılığının Karma Rasch Modelleri İle İncelenmesi, PhD thesis,,Ankara 